\title{
Management of primary adenocarcinoma of the female urethra: Report of two cases and review of the literature
}

\author{
XINJUN WANG ${ }^{1,2}$, PEIMING BAI ${ }^{2}$, HANZHONG SU $^{2}$, GUANGCHENG LUO ${ }^{2}$, \\ ZHAOHUI ZHONG ${ }^{1}$ and XIAOKUN ZHAO ${ }^{1}$ \\ ${ }^{1}$ Department of Urology, The Second Xiangya Hospital, Central South University, Changsha, Hunan 410011; \\ ${ }^{2}$ Department of Urology, Zhongshan Hospital, Xiamen University, Xiamen, Fujian 361004, P.R. China
}

Received April 30, 2012; Accepted August 22, 2012

DOI: $10.3892 / \mathrm{ol} .2012 .886$

\begin{abstract}
Primary adenocarcinoma of the female urethra is rare and only a few retrospective cases have been published. The origin of urethral adenocarcinomas remains unclear. Certain authors have suggested that urethral adenocarcinomas in females originate at the periurethral Skene's glands. We report one case of urethral adenocarcinoma of the proximal urethra in a 44-year-old female who presented with painless urethral bleeding. Abdominal and pelvic CT scan with contrast and chest radiology were unremarkable. Biopsy of the mass revealed adenocarcinoma of the urethra. The patient was treated with partial urethrectomy and was free of disease for more than 5 years. We also present another rare case of mucinous adenocarcinoma in a 52-year-old female who complained of an enlarged urethral mass. Pelvic MRI revealed a tumor surrounding the whole urethra and bilateral inguinal lymph nodes. A puncture biopsy later revealed that the tumor was mucinous adenocarcinoma. Anterior pelvic exenteration with pelvic and bilateral lymph node dissection was performed and chemotherapy was administered. The patient was followed up for 12 months and did not experience local recurrence or distant metastasis. In conclusion, for the diagnosis of urethral cancer, a biopsy is necessary for any suspicious urethral lesions. MRI is recommended for tumor staging. Small, superficial, distal urethral tumors may be treated with excision of the distal urethra. For advanced female urethral cancer, a combination of chemotherapy, radiation therapy and surgery is recommended for optimal local and distant disease control. Regular follow-up is required in these patients.
\end{abstract}

Correspondence to: Professor Xiaokun Zhao, Department of Urology, The Second Xiangya Hospital, Central South University, No. 139 Renmin Road, Changsha, Hunan 410011, P.R. China E-mail: xiaokunzhao@163.com

Key words: female urethral cancer, mucinous adenocarcinoma, surgery

\section{Introduction}

Female urethral carcinoma is rare and accounts for approximately $0.02 \%$ of all female cancers and less than $1 \%$ of cancers in the female genitourinary tract (1). In females, squamous cell carcinoma is the most common histological type, accounting for $70 \%$ of all cases. Transitional cell carcinoma (20\%) and adenocarcinomas (8-10\%) are the next most common cell types (2). Most patients are symptomatic at presentation. Certain patients may present with obstructive symptoms, dysuria, urethral bleeding, urinary frequency and often a palpable urethral mass or induration. The evaluation of females with suspected urethral carcinoma includes cystourethroscopy, physical examination, CT and MRI of the abdomen and pelvis and chest radiography. The prognosis is determined largely by the clinical stage and the location of the lesions. Tumors in the distal urethra tend to have a better outcome (3).

Primary adenocarcinoma of the female urethra is extremely rare with only a few retrospective cases published. We report two cases of female urethral adenocarcinoma, including a rare case of mucinous adenocarcinoma. The study was approved by the ethics committee of Zhongshan Hospital, Xiamen University, China. Informed consent was obtained from each patient.

\section{Case 1}

A 44-year-old female patient complained of painless occasional urethral bleeding over the previous 2 months. Physical examination revealed a reddish hemispheroid mass measuring $15 \mathrm{~mm}$ in diameter without bleeding or tenderness at the posterior lip of the urethral meatus. No enlarged inguinal lymph nodes were identified. Serum studies were normal and urinalysis revealed numerous red blood cells per high power field. Transvaginal ultrasonography of the urethra revealed a well-defined hyperechoic mass with abundant blood flow, with approximate dimensions of 12x11x10 mm (Fig. 1). Abdominal and pelvic CT scan with contrast and chest rediology was unremarkable. Biopsy of the mass revealed adenocarcinoma of the urethra. The patient underwent partial urethectomy and frozen-section pathology was performed to ensure an adequate margin. Subsequent routine pathology reported adenocarci- 


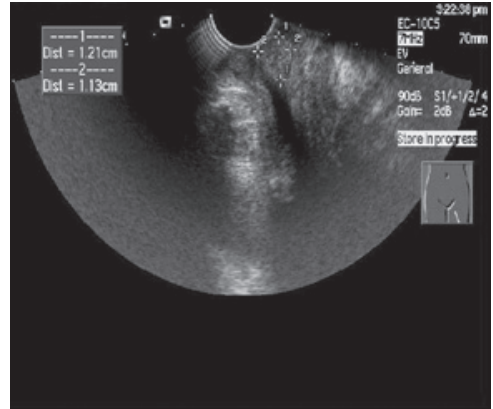

Figure 1. Transvaginal ultrasonography of urethra demonstrating a welldefined hyperechoic mass with approximate dimensions of 12x11x10 mm.

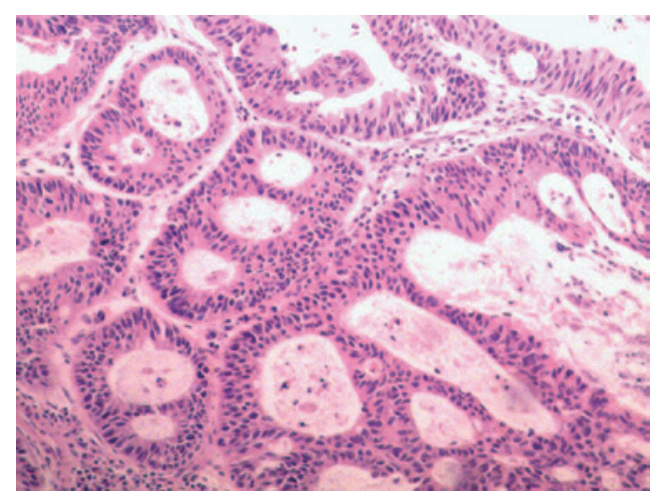

Figure 2. HE, x100. Adenocarcinoma of distal female urethra.

noma of the urethra with a free margin consistent with the previous pathology report (Fig. 2). The patient recovered without complication and retained normal void function. The patient was followed up for more than 5 years and remained free of local recurrence and distant metastasis.

\section{Case 2}

A 52-year-old female patient was admitted for a gradually enlarging urethral mass with itching over the past 3 years. The patient developed acute urinary retention 10 days before admission, therefore a 16-French urinary catheter was inserted in a local hospital. The patient had undergone tubal lignations 20 years earlier. Vaginal examination revealed a fixed hard mass measuring approximately $3 \times 2 \times 3 \mathrm{~cm}$ beneath the anterior vaginal wall, without tenderness and bleeding. Two enlarged lymph nodes $2.5 \mathrm{~cm}$ in diameter were felt in the bilateral inguinal regions, which were hard and mobilized but free of tenderness. Pelvic MRI with contrast revealed a $5 \times 2.9 \times 3.6-\mathrm{cm}$ tumor surrounding the urethra, with slightly higher signal in T1- and T2-weighted images and uneven enhancement after contrast administration (Fig. 3A and B). Enlarged lymph nodes with even enhancement measuring approximately $1.8 \times 2.6 \mathrm{~cm}$ were observed in the bilateral inguinal region (Fig. 3C). The results of routine hematology studies and chemistry blood tests were in the normal range. Tumor serum markers, including carcinoembryonic antigen, $\alpha$-fetoprotein and prostate-specific antigen (PSA), were all in the normal range. Cystourethroscopy revealed normal urethral and bladder mucous membrane with some urethral
A

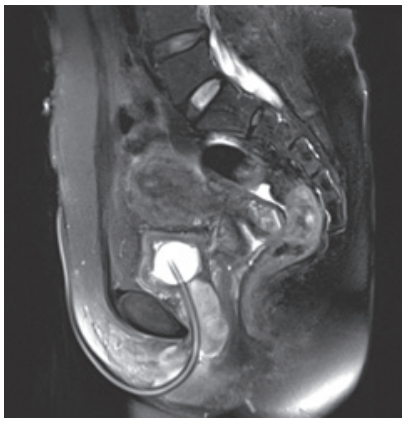

B

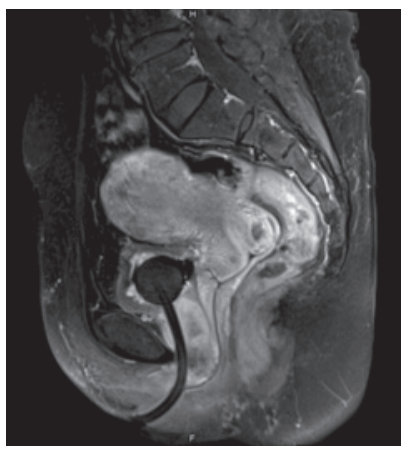

C

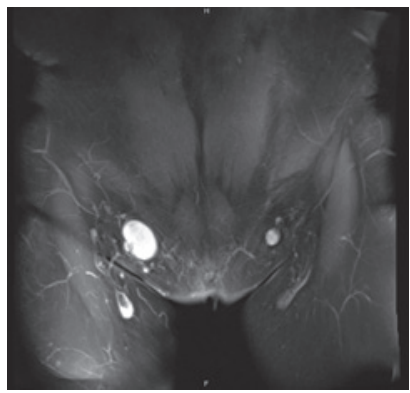

Figure 3. Pelvic MRI demonstrating lesion surrounding the urethra, (A) with slightly higher signal in T1- and T2-weighted images and (B) uneven enhancement after contrast administration. (C) Enlarged lymph nodes with even enhancement were observed in the bilateral inguinal region.

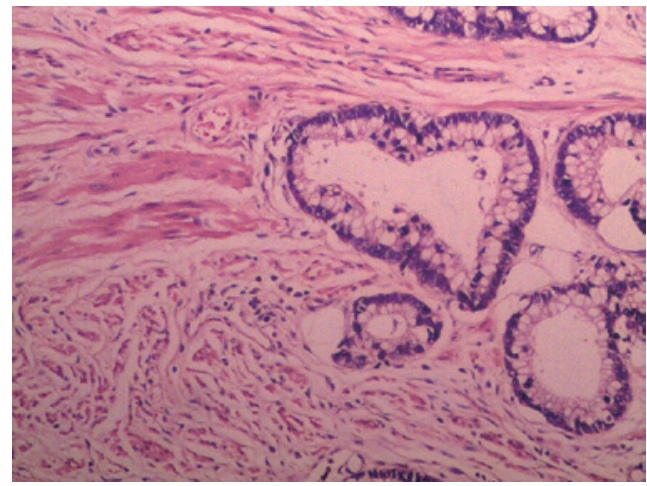

Figure 4. HE, x100 showing mucinous adenocarcinoma of urethra with invasion to the superficial muscular layer of the anterior vaginal wall.

hyperemia. Biopsy of the urethral membrane revealed chronic inflammation of the urethra. A puncture biopsy later revealed mucinous adenocarcinoma of the tissues between the urethra and anterior vaginal wall with a possibility of a metastatic lesion from the intestinal tract or ovaries. However, the clinical data did not support the hypothesis that the lesion was metastatic, as pelvic MRI revealed normal ovaries and 
metastatic screening, including chest radiology, ultrasonography of the abdomen and nuclide bone scan, and serum tumor markers were unremarkable.

After careful preoperative preparation, the patient underwent anterior pelvic exenteration, including anterior vaginal wall, urethra, bladder and uterus, with pelvic and bilateral lymph node dissection and ileum conduit. Postoperative pathology reported moderately differentiated mucinous adenocarcinoma of the posterior urethra, including bladder neck, internal urethral orifice and external urethral orifice. The tumor had invaded the superficial muscular layer of the anterior vaginal wall, but the vaginal mucosa was not affected (Fig. 4). Metastases were present in the right pelvic lymph nodes (1/3), left inguinal lymph nodes (1/9) and the right inguinal lymph nodes (2/9). The patient recovered gradually in 4 weeks and healed satisfactorily. The patient was then transferred to the Department of Oncology, Zhongshan Hospital, Xiamen University, Fujian, China, and accepted two courses of chemotherapy regimen of gemcitabine plus cisplatin. The patient was followed up for 12 months without local recurrence or distant metastasis. Lymphedema in the left lower extremity was present 2 months postoperatively and resolved after medical management and using elastic socks. The ileum conduit functioned well.

\section{Discussion}

Female urethral carcinoma is among the rarest types of neoplasia of the genitourinary tract and corresponds to $0.003 \%$ of all malignant neoplasias occurring in the female urogenital tract (4). Although it was once believed that urethral cancer was four times more common in females than males, more recent literature suggests that primary urethral cancer is nearly three times more common in males: a Surveillance, Epidemiology and End Results (SEER) study reported an incidence of 4.3 per million in males and 1.5 per million in females (5).

In females, squamous cell carcinoma is the most common histological type of urethral carcinoma, accounting for $70 \%$ of all cases. Transitional cell carcinoma (20\%) and adenocarcinomas (8-10\%) are the next most common cell types. Other rarer cell types include lymphoma, neuroendocrine carcinoma, sarcomas, paragangliomas, melanoma and metastasis (2).

Mucinous adenocarcinoma is most often composed of colonic-type glandular epithelium and may contain abundant extracellular mucin; it resembles mucinous carcinoma of the colon and rectum, although it may occur at other sites, including the pancreas, stomach, prostate and breast (6). Mucinous adenocarcinoma of the female urethra is rare, with 25 cases reported in the English literature (7-9) and one case report in the Japanese literature (10).

Etiological factors associated with the development of urethral carcinoma in females include leukoplakia, chronic irritation, caruncles, polyps, parturition and human papillomavirus infection or other viral infections. Female urethral diverticula may also predispose the patient to malignant change and adenocarcinoma accounts for more than $60 \%$ of female urethral carcinomas arising within a diverticulum (11).

The origin of urethral adenocarcinomas remains unclear. Certain authors have suggested that urethral adenocarcinomas in females originate at the periurethral Skene's glands, which is a homolog of the prostate. PSA positivity is considered to be evidence for an origin from the Skene's glands (12). Reis et al reported that the macroscopic and microscopic examination of two PSA-negative adenocarcinomas, including cytochemical and immunohistochemical studies, favored an origin from Skene's glands due to similarities to findings in normal Skene's glands $(3,13)$. Chan et al reported a case suggesting that mucinous urethral adenocarcinoma may arise from the malignant transformation of urethritis glandularis (13).

Most patients with urethral carcinoma are symptomatic at presentation. A number of patients present with obstructive symptoms, dysuria, urethral bleeding, urinary frequency and often a palpable urethral mass or induration. A suspicion of a urethral tumor should be raised in any otherwise healthy middle-aged female without prior urological history who presents with urinary retention. Patients may also present with a small lesion prolapsing through the urethral meatus or with a small submucosal lesion on the anterior wall of the vagina. Tumors spread typically by local extension and may ulcerate as the tumor progresses to the skin and vulvar region. Proximal lesions may extend posteriorly into the vagina or proximally into the bladder. Lymphatic spread is uncommon at early stages, but clinically palpable nodes may be present in up to one-third of patients at presentation and half of patients with advanced and proximal tumors. Hematogenous spread may occur to the lung, liver, bone and brain, in order of frequency (14).

The evaluation of females with suspected urethral carcinoma includes cystourethroscopy, physical examination under anesthesia, computed tomography of the abdomen and pelvis and chest radiography. MRI has been used to evaluate pelvic lesions and may aid the determination of local extension. MRI has been reported to be accurate for evaluating local urethral tumors in $90 \%$ of patients. Urethral tumors typically appear hypointense on T1-weighted images and relatively hyperintense on T2-weighted images. Tumor extent is best evaluated on sagittal T2-weighted images. Tumors in the distal urethra may extend into the adjacent perineum and the target-like appearance of the normal urethra on axial T2-weighted images may be disrupted (15). CT may reveal a urethral mass with soft-tissue attenuation (16). Transvaginal ultrasonograpy may also provide clues for diagnosis. Biopsy for suspected tumor is essential for establishing the diagnosis. For the diagnosis of primary urethral mucinous adenocarcinoma, exclusion of metastatic lesion originating from the intestinal tract or ovaries is essential.

Due to the rarity of this type of tumor and heterogeneity of the disease, most studies report similar prognoses in different histological subtypes $(17,18)$. Certain studies have suggested that squamous cell carcinoma tends to have a lower recurrence rate compared with adenocarcinoma and transitional cell carcinoma, but the case series are too small to have any statistical significance $(17,18)$. The prognosis is determined largely by the clinical stages and the location of the lesions. Tumors in the distal urethra tend to have a better outcome (3).

Options for treatment of female urethral carcinoma include surgery, radiation therapy and chemotherapy, alone or in combination. Treatment has tended toward a multimodality approach in recent years. Local excision, which should lead to excellent functional results, may be sufficient for the relatively 
uncommon small, superficial, distal urethral tumors. Proximal female urethral carcinomas are more likely to be high stage and may extend into the bladder and vagina, as in the second case described in the present study. For advanced female urethral cancer, a combination of chemotherapy, radiation therapy and surgery has been recommended for optimal local and distant disease control (19). Due to the high morbidity associated with inguinal dissection and lack of improved survival, inguinal dissection is not routinely performed and is only for patients who present with positive inguinal or pelvic lymphadenopathy without distant metastasis or those who develop regional lymphadenopathy during surveillance (14).

Primary adenocarcinoma of female urethra is rare. A biopsy is necessary for any suspicious urethral lesions. MRI is recommended for tumor staging. Small, superficial, distal urethral tumors may be treated with excision of the distal urethra. For advanced female urethral cancer, a combination of chemotherapy, radiation therapy and surgery is recommended for optimal local and distant disease control. Regular follow-up is required in these patients.

\section{References}

1. Srinivas V and Khan SA: Female urethral cancer - an overview. Int Urol Nephrol 19: 423-427, 1987.

2. Ouzaid I, Hermieu JF, Dominique S, Fernandez P, Choudat L and Ravery V: Management of adenocarcinoma of the female urethra: case report and brief review. Can J Urol 17: 5404-5407, 2010.

3. Gheiler EL, Tefilli MV, Tiguert R, de Oliveira JG, Pontes JE and Wood DP Jr: Management of primary urethral cancer. Urology 52: 487-493, 1998.

4. Reis LO, Billis A, Ferreira FT, Ikari LY, Stellini RF and Ferreira U: Female urethral carcinoma: evidences to origin from Skene's glands. Urol Oncol 29: 218-223, 2011.

5. Swartz MA, Porter MP, Lin DW and Weiss NS: Incidence of primary urethral carcinoma in the United States. Urology 68: 1164-1168, 2006

6. Chen YB and Epstein JI: Primary carcinoid tumors of the urinary bladder and prostatic urethra: a clinicopathologic study of 6 cases. Am J Surg Pathol 35: 442-446, 2011.
7. Ney C, Miller HL and Ochs D: Adenocarcinoma in a diverticulum of the female urethra: a case report of mucous adenocarcinoma with a summary of the literature. J Urol 106: 874-877, 1971.

8. Meis JM, Ayala AG and Johnson DE: Adenocarcinoma of the urethra in women. A clinicopathologic study. Cancer 60: 1038-1052, 1987.

9. Murphy DP, Pantuck AJ, Amenta PS, et al: Female urethral adenocarcinoma: immunohistochemical evidence of more than 1 tissue of origin. J Urol 161: 1881-1884, 1999.

10. TanakaH,MasudaH,KomaiY,etal:Primaryadenocarcinoma of the female urethra treated by multimodal therapy. Hinyokika Kiyo 55: 43-46, 2009 (In Japanese).

11. Awakura Y, Nonomura M, Itoh N, Maeno A and Fukuyama T: Adenocarcinoma of the female urethral diverticulum treated by multimodality therapy. Int J Urol 10: 281-283, 2003.

12. Dodson MK, Cliby WA, Keeney GL, Peterson MF and Podratz KC: Skene's gland adenocarcinoma with increased serum level of prostate-specific antigen. Gynecol Oncol 55: 304-307, 1994

13. Chan YM, Ka-Leung Cheng D, Nga-Yin Cheung A, Yuen-Sheung Ngan $\mathrm{H}$ and Wong LC: Female urethral adenocarcinoma arising from urethritis glandularis. Gynecol Oncol 79: 511-514, 2000.

14. Karnes RJ, Breau RH and Lightner DJ: Surgery for urethral cancer. Urol Clin North Am 37: 445-457, 2010.

15. Gourtsoyianni S, Hudolin T, Sala E, Goldman D, Bochner BH and Hricak H: MRI at the completion of chemoradiotherapy can accurately evaluate the extent of disease in women with advanced urethral carcinoma undergoing anterior pelvic exenteration. Clin Radiol 66: 1072-1078, 2011

16. Kawashima A, Sandler CM, Wasserman NF, LeRoy AJ, King BF Jr and Goldman SM: Imaging of urethral disease: a pictorial review. Radiographics 24 (Suppl 1): S195-S216, 2004.

17. Dimarco DS, Dimarco CS, Zincke H, et al: Surgical treatment for local control of female urethral carcinoma. Urol Oncol 22: 404-409, 2004.

18. Foens CS, Hussey DH, Staples JJ, Doornbos JF, Wen BC and Vigliotti AP: A comparison of the roles of surgery and radiation therapy in the management of carcinoma of the female urethra. Int J Radiat Oncol Biol Phys 21: 961-968, 1991.

19. Wein AJ KL, Novick AC, Partin AW and Peters CA (eds): Campbell-Walsh Urology. Vol 4. 10th edition. Saunders Elsevier, Philadelphia, PA, 2011. 